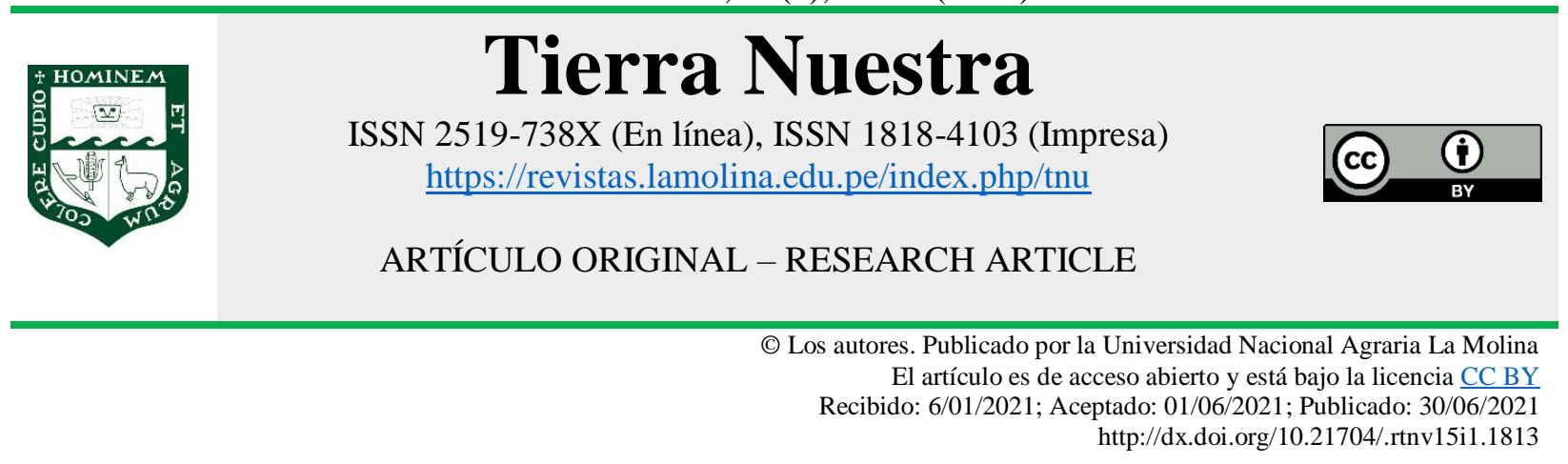

\title{
Fundamentos filosóficos, ideológicos en la Política Educativa
}

\section{Philosophical, ideological foundations in Educational Policy}

\author{
Marilyn Aurora Buendía Molina 1,2*(D), Elva María Ríos Ríos ${ }^{1,2 *(D)}$, Angela Patricia Salazar \\ Villar $^{2 *(D)}$, Rosario Milagros Allcca Sovero ${ }^{2 *}$ (iD \\ * Autor de correspondencia: marilynbuendia@lamolina.edu.pe
}

\section{RESUMEN}

La presente investigación tiene como objetivo describir y analizar los fundamentos filosóficos e ideológicos en la Política educativa, a partir de fuentes primarias de artículos publicados entre el 2016 al 2021. Se utilizó una metodología de enfoque cualitativo y diseño de investigación de teoría fundamentada tomando proposiciones teóricas de investigaciones. El método utilizado fue de revisión sistemática de tipo documental, crítico-creativo y hermenéutico, la unidad de análisis fueron estudios originales primarios, los cuales permitieron sintetizar información disponible con respecto a los fundamentos filosóficos e ideológicos en la política educativa. Como resultante mostramos diferentes interpretaciones los cuáles permitieron formular propuestas para el mejoramiento de las políticas educativas desde la filosofía e ideología educativa. Se concluye que tanto la filosofía como la ideología son pilares imprescindibles para el desarrollo de la política educativa de cualquier país, son la herramienta que tienen los gobiernos para involucrarse en la forma en el cual se producen y distribuyen los conocimientos en una sociedad.

Palabra clave: ideología, filosofía, política educativa, educación, inclusión.

\section{ABSTRACT}

The objective of this research is to describe and analyze the philosophical and ideological foundations in Educational Policy, from primary sources of articles published between 2016 and 2021. A qualitative approach methodology and grounded theory research design were used, taking theoretical propositions of investigations. The method used was a systematic review of the documentary, critical-creative and hermeneutic type, the unit of analysis was primary studies, which allowed us to synthesize available information regarding the philosophical and ideological foundations in educational policy. The population and sample consisted of nine articles. As they turned out, we showed different interpretations, the results allowed to formulate proposals for the improvement of educational policies from the educational philosophy and ideology. It is concluded that both philosophy and

Forma de citar el artículo (Formato APA):

Buendía, M., Ríos, E., Salazar, A., \& Allcca, R. (2021). Fundamentos filosóficos, ideológicos en la Política Educativa. Tierra Nuestra. 15(1), 29-35. http://dx.doi.org/10.21704/rtn.v15i1.1813

${ }^{1}$ Universidad Nacional Agraria La Molina, 15024, Lima, Perú. marilynbuendia@lamolina.edu.pe; erios@lamolina.edu.pe

${ }^{2}$ Universidad Nacional de Educación Enrique Guzmán y Valle. rallccas@gmail.com; angela.salazar@upn.edu.pe 
ideology are essential pillars for the development of education in any country, they are the political tool that governments have to get involved in the way in which knowledge is produced and distributed in a society.

Keywords: ideology, philosophy, educational policy, education, inclusion.

\section{Introducción}

El estudio de la educación es complejo, por implicar en su integridad al ser humano; es decir, con su estructura biopsicosocial y espiritual. Morin (2002) determina que los fundamentos son las bases que dan solidez y seguridad a lo que se pretende construir.

Lewin (1988) afirma que "la mejor práctica es una buena teoría", pero no se debe desconocer la existencia de otros tipos de fundamentos como los económicos, psicológicos, sociológicos, entre otros. Siendo los filosóficos los más importantes para el diseño de políticas educativas, porque los procesos educativos demandan de sus actores pensar bien, reflexionar y actuar en forma coherente. En ese sentido, las políticas educativas son acciones que tienen lugar en un sistema educativo, teniendo por propósito el conjunto de instituciones preparar al ser humano para la vida adulta.

Para lograr una verdadera formación del ser humano, en la línea de la antropología filosófica esbozada, se toma al hombre como un conjunto y núcleo de intencionalidades para desarrollar esas potencialidades y obtener un ser humano realizado y pleno, de modo que pueda repetir y reproducir ese modelo en su acción y, si es maestro, también en su enseñanza a los estudiantes. Es un paradigma y modelo de educación, que tiene que ser puesto en la práctica con aditamentos técnicos, pero antes que nada con principios filosóficos Beuchot (2015).

\section{Marco teórico}

Tenemos a Morales-Gómez et al. (2019) quienes en su investigación analizaron los fundamentos filosóficos en los que debería cimentarse un modelo educativo de calidad. Cuyos resultados fueron: auscultar $\mathrm{y}$ profundizar en el sentido y el alcance de lo que representa la definición de fundamentos filosóficos aplicados a una propuesta educativa innovadora. Además, los fundamentos filosóficos de un modelo educativo de calidad tienen que ver con los siguientes elementos: principios, fines, criterios, premisas, valores, conceptos y contextos. Siendo los fines los que contienen la filosofía y los contextos demarcan los entornos reales en los que se forman los sujetos de aprendizaje, destinatarios directos de dichos proyectos. Posteriormente, Morales-Gómez et al. (2019) reportan que los fundamentos filosóficos y los fundamentos antropológicos de la educación están estrechamente unidas, porque en la educación está implicado el ser humano completo, con su estructura biopsicosocial, cultural y espiritual. Es importante recalcar que los fundamentos son los cimientos que dan seguridad a lo que el ser humano pretende construir. De ahí que, se debe tener buenos fundamentos que garanticen que lo construido se pueda mantener en el tiempo.

Por otro lado, Tello (2019) en "Notas Históricas y Epistemológicas Sobre El Campo de La Política Educativa En Latinoamérica" señala que existen múltiples epistemologías de la política educativa, sin embargo se debe tener en cuenta que en la producción de conocimiento de cada investigador se tendría que poder observar una epistemología coherente y sólida con los tres componentes que las conforman: posicionamiento epistemológico, es la teoría sustantiva (Glaser y Strauss, 1967) vinculada especialmente al campo de estudio, a las corrientes teóricas del campo, considerando que las teorías sustantivas son las que guardan una relación directa con el contenido empírico y teórico de los datos de la investigación; perspectiva Epistemológica, en cada una de las investigaciones en política educativa, la cosmovisión que el investigador asume para guiar el estudio, Glaser y Strauss (1967) lo ha denominado la teoría general. Debemos considerar que no existe una "cabeza vacía" en términos epistemológicos, sin embargo, existen "cabezas abiertas" en búsqueda de resultados de investigación; y, enfoque Epistemetodológico. Además, consideran que la investigación en política educativa requiere una transformación epistemológica, tanto en las ciencias sociales como en las ciencias políticas, una 
transformación epistemológica que sea capaz de desafiar las grandes verdades establecidas para establecer la política educativa. En conclusión, Tello (2019) señala que la investigación en políticas educativas contribuye a fortalecer y desarrollar la justicia educativa y la consolidación democrática en Latinoamérica. Asimismo, Compañ (2020) en la publicación intitulada "Acciones de políticas educativas ante la emergencia sanitaria del Covid-19" logró describir y analizar las acciones de política educativa diseñadas e implementadas por la administración del gobierno de México ante las condiciones de pandemia Covid 19. La política educativa fue plantear la educación a distancia con el uso de diversos recursos tecnológicos. Sin embargo, la política educativa no es equitativa ni inclusiva, debido que un amplio sector de la población no ha podido ser atendida y se mantienen excluidos de tal beneficio. Antes de establecer una política educativa se debe tener en cuenta la ideología los fundamentos ideológicos de una sociedad. Tal como se puede apreciar en la publicación "Lo histórico y filosófico Fundamentos del conservador Política educativa" de Percival (2017) donde la política educativa conservadora contemporánea se basa en creencias ideológicas profundamente arraigadas.

\section{Métodos}

El presente artículo de revisión sistemática es de tipo documental, crítico-creativo y hermenéutico. Documental, porque se ha basado en la consulta $y$ revisión de distintas fuentes bibliográficas, tanto filosóficas, epistemológicas como educativas. Crítico- creativo, por cuanto se han abierto caminos para la reflexión, el diálogo y análisis en materia de política educativa. Hermenéutico, porque la interpretación de textos, corrientes e ideologías ha permitido clarificar el pensamiento en la búsqueda y construcción de los fundamentos filosóficos e ideológicos en la política educativa.

La población fueron 20 manuscritos que se encuentran en bases de datos Scielo, Dialnet y Latindex, mientras que nueve manuscritos fueron los artículos seleccionados por su contenido.

El problema que motivo la investigación fue la identificación de los fundamentos filosóficos, ideológicos en la política educativa, la cual fue estudiada bajo diferentes enfoques e investigaciones con el propósito de establecer políticas educativas acorde a la necesidad de cada realidad.

La pregunta de la investigación fue ¿cuáles son los fundamentos filosóficos, ideológicos en la política educativa, qué ideas asumen acerca de la persona humana, de la sociedad y del acto educativo, y de las relaciones entre las tres?

\section{Resultados}

Los artículos revisados posibilitaron sintetizar retos planteados por los autores a raíz de las definiciones que permitieron sugerir alcances sobre fundamentos Filosóficos e Ideológicos en la Política Educativa (Tabla 1).

Tabla 1. Fundamentos filosóficos e ideológicos en la Política Educativa

\begin{tabular}{|l|l|}
\hline \multicolumn{1}{|c|}{ Autores } & \multicolumn{1}{c|}{ Fundamentos } \\
\hline $\begin{array}{l}\text { Morales-Gómez et al. } \\
\text { 2019, p. 18) }\end{array}$ & $\begin{array}{l}\text { Las filosofías de la educación son las maneras plurales de concebir y realizar el acto de } \\
\text { educar. El filosofar es un ejercicio mental de reflexión sobre realidades fácticas } \\
\text { existenciales (cosas, sucesos, personas, etc.) y dentro de ellas se encuentra la educación, } \\
\text { que trata de teorías y métodos e interrelaciones humanas. }\end{array}$ \\
\hline $\begin{array}{l}\text { Morales-Gómez et al. } \\
\text { 2019, p. 118) }\end{array}$ & $\begin{array}{l}\text { En el credo pedagógico de Dewey encontramos tres palabras claves para entender el papel } \\
\text { de la Filosofía en la Educación, a saber: comprensión, interpretación, significación. La } \\
\text { comprensión nos remite a la reflexión, la interpretación a la crítica y la significación al } \\
\text { sentido. }\end{array}$ \\
\hline
\end{tabular}




\begin{tabular}{|c|c|}
\hline $\begin{array}{l}\text { Osho (1993) citado } \\
\text { en Morales-Gómez } \\
\text { et al. (2019) }\end{array}$ & $\begin{array}{l}\text { La Filosofía de la Educación con la ayuda de la Antropología Filosófica, busca integrar } \\
\text { los saberes sobre el ser humano en una síntesis coherente y comprensiva, que le permitan } \\
\text { a éste "florecer desde su propia naturaleza intrínseca" }\end{array}$ \\
\hline Fernández (2016) & $\begin{array}{l}\text { "Cuando hablamos de fundamentos filosóficos en el orden educativo, hay que referirse de } \\
\text { manera obligada a la Filosofía de la Educación, que trata acerca del quehacer educativo, } \\
\text { pero no de forma abstracta sino a partir de la propia práctica educativa". }\end{array}$ \\
\hline $\begin{array}{l}\text { Fernández (2016), p. } \\
\text { 117) }\end{array}$ & $\begin{array}{l}\text { El objeto de la Filosofía de la Educación cuando se trata de Educación Inclusiva y atención } \\
\text { a la diversidad hay que concretarlo en problemas relacionados que atañan a dicho proceso } \\
\text { y que conllevan a la no exclusión". }\end{array}$ \\
\hline Fernández (2016) & $\begin{array}{l}\text { "La Filosofía de la Educación, es un fundamento básico de las ideas educativas y } \\
\text { pedagógicas y constituye referencia obligada de todos los profesores, directivos y asesores, } \\
\text { como única vía para apreciar los modelos educativos que rigen en las educaciones". }\end{array}$ \\
\hline $\begin{array}{l}\text { Martínez y Rodríguez } \\
(2002) \text { citado por } \\
\text { Fernández (2016), p. } \\
\text { 8) }\end{array}$ & $\begin{array}{l}\text { "Se asume como Filosofía de la Educación: Un saber general de los procesos y acciones } \\
\text { educativas a partir de presupuestos antropológicos, epistemológicos, axiológicos con un } \\
\text { enfoque crítico metodológico general o como una concepción general del proceso } \\
\text { educativo que surge como resultado de la aplicación de un enfoque integrador, } \\
\text { cosmovisivo y crítico axiológico de este proceso complejo y contradictorio en que se } \\
\text { produce la formación del hombre mediante la asunción creadora de los valores de la } \\
\text { cultura universal a partir de la identidad nacional y de la transformación práctica". }\end{array}$ \\
\hline $\begin{array}{l}\text { Martínez y Rodríguez } \\
\text { (2002) citado por } \\
\text { Fernández (2016) }\end{array}$ & $\begin{array}{l}\text { "Reflexionar sobre el sentido de la educación es función básica de la Filosofía de la } \\
\text { Educación por tanto esa reflexión se vuelve infinita en tanto lo es así el desarrollo } \\
\text { humano". }\end{array}$ \\
\hline $\begin{array}{l}\text { Medina, Barrio, } \\
\text { Cisneros (2016)) }\end{array}$ & $\begin{array}{l}\text { "Resulta, altamente difícil plantear una educación inclusiva sin antes realizar una revisión } \\
\text { de diversas concepciones sociológicas y filosóficas, siendo imprescindible revisar ciertos } \\
\text { aspectos de la idea predominante sobre la educación". }\end{array}$ \\
\hline $\begin{array}{l}\text { Inés de la C. Valdés, } \\
2011 \text { citado por } \\
\text { Medina et al. (2016) }\end{array}$ & $\begin{array}{l}\text { "La educación toma de la filosofía la reflexión y el punto de vista de la totalidad para } \\
\text { esclarecer los problemas relativos a la pedagogía. Obteniendo el educador una mayor } \\
\text { conciencia de su labor educativa y saber que su práctica descansa sobre temas que se } \\
\text { imbrican con la humanidad y su práctica más inmediata". }\end{array}$ \\
\hline Pérez (2018) & $\begin{array}{l}\text { Es necesario diseñar e implementar un currículo, desde la perspectiva no sexista, que } \\
\text { forme a hombres y mujeres para que se perciban y se relacionen como iguales. Desde las } \\
\text { instituciones universitarias se deben posibilitar algunas estrategias prácticas que permitan } \\
\text { incorporar la perspectiva de género en la elaboración de guías docentes y en el diseño de } \\
\text { actividades en el aula. Los docentes deben analizar todas las implicaciones del proceso de } \\
\text { aprendizaje para plantear propuestas de innovación que ayuden al estudiantado a ser } \\
\text { competentes para enfrentarse al aprendizaje en su formación integral, y se comprometan a } \\
\text { promover la igualdad de género. }\end{array}$ \\
\hline $\begin{array}{l}\text { Muñoz, } \quad \text { Lobos, } \\
\text { Kawada, \& } \text { Buzzetti } \\
(2020)\end{array}$ & $\begin{array}{l}\text { La documentación analizada reconoce y señala el valor del individuo en su especificidad } \\
\text { cultural y de origen, considerando su lengua, cosmovisión e historia, tal como lo señala la } \\
\text { Ley General de Educación: "la diversidad cultural, religiosa y social de las poblaciones } \\
\text { que son atendidas" (Ley } 20.370,2009 \text {, p. 2). Sin embargo, las diversidades culturales, } \\
\text { religiosas y sociales no son certificables como Necesidades Educativas Especiales; por } \\
\text { tanto, es reconocida su presencia, pero no reestructuran al sistema educativo, no son en } \\
\text { propiedad tratadas como otredades legítimas y en igualdad de derechos. }\end{array}$ \\
\hline Muñoz et al. (2020) & $\begin{array}{l}\text {...se percibe que la escuela adopta y reproduce el relato de espacio de construcción de } \\
\text { sociedades más equitativas y justas; no obstante, en su actuar no realiza una } \\
\text { reestructuración sobre lo que se entiende por diversidad, sino que se limita al } \\
\text { reconocimiento de algunas condiciones de exclusión, siendo además particular que los } \\
\text { discursos de inclusión son siempre dirigidos a los alumnos como objetivo, invisibilizando } \\
\text { y excluyendo las diversidades que se encuentran en el profesorado. }\end{array}$ \\
\hline Tello (2019) & $\begin{array}{l}\text { Existen múltiples epistemologías de la política educativa, sin embargo, se debe tener en } \\
\text { cuenta que en la producción de conocimiento de cada investigador se tendría que poder }\end{array}$ \\
\hline
\end{tabular}




\begin{tabular}{|l|l|}
\hline & $\begin{array}{l}\text { observar una epistemología coherente y sólida con los tres componentes que las } \\
\text { conforman: Posicionamiento epistemológico, Perspectiva Epistemológica y Enfoque } \\
\text { Epistemetodológico. }\end{array}$ \\
\hline Tello (2019) & $\begin{array}{l}\text { Consideramos que la investigación en política educativa requiere una transformación } \\
\text { epistemológica, como se reclama desde las ciencias sociales y las ciencias políticas, una } \\
\text { transformación epistemológica que sea capaz de desafiar las grandes verdades establecidas } \\
\text { y desde la investigación repensar la política educativa. }\end{array}$ \\
\hline
\end{tabular}

\section{Discusión}

Existen diversas interpretaciones sobre Fundamentos Filosóficos en Política Educativa, todos los aportes revisados coinciden en que, los fundamentos tienen que ver con la comprensión de la actividad educativa. Y todo esto con el fin de lograr la inserción activa y eficaz de un individuo en la sociedad, que le permita una convivencia armónica con sus semejantes. Eso es precisamente lo que señaló John Dewey (1987) en su conocido Credo Pedagógico: comprensión, interpretación, significación. La comprensión nos remite a la reflexión, la interpretación a la crítica y la significación al sentido.

Las filosofías de la educación son las maneras plurales de concebir y realizar el acto de educar. El filosofar es un ejercicio mental de reflexión sobre realidades fácticas existenciales (cosas, sucesos, personas, etc.) y dentro de ellas se encuentra la educación, que trata de teorías y métodos e interrelaciones humanas.

Existen diversas interpretaciones sobre Política Educativa, todos los aportes revisados coinciden en que la Política Educativa tiene fundamentos Filosóficos e ideológicos. Para Martínez (2018) las políticas educativas es el conjunto de instituciones cuyo propósito es preparar a niños y adolescentes para la vida adulta. En las sociedades de la antigüedad no era necesario los estudios especializados; pero con el pasar de los años las sociedades se han vuelto complejas y con ello surgen las instituciones educativas. Mientras que, Viennet y Pont (2017) definen la política educativa como las acciones emprendidas por un gobierno en relación con prácticas educativas y la forma en que el gobierno atiende la producción y oferta de la educación. Algunos entienden por política educativa, el reconocer que algunos actores privados u otras instituciones, organismos internacionales y ONG's pueden organizar políticas educativas, otra definición de Política Educativa es dada por Rayou y Van Zanten (2015):
"Las políticas educativas son programas desarrollados por las autoridades públicas, informados por valores e ideas, dirigidos a actores de la educación, e implementados por administradores y profesionales de la educación". Asimismo, El éxito de las políticas educativas es en base a la situación actual de acuerdo a lo que se necesita. En ese sentido Compañ (2020) brinda algunas pistas que puedan cooperar a construir políticas educativas efectivas. A) Política visionaria: la política educativa debe considerar el contenido y su articulación con su proceso de formulación. B) Política basada en la cooperación: es una política educativa que se enmarca en un camino intencional donde la cooperación es un elemento estratégico importante y necesario. Y, C) Política de los contextos: la política educativa parte sobre la atención a una población diversa y diferente. A través de estrategias homogéneas y de asimilación que genera costos importantes a favor de lo más desfavorecidos. Finalmente, la Política Educativa se implementa una vez que se ha formulado los principales propósitos en cuanto a las acciones de política educativa, también se debe impulsar varias estrategias para lograr la implantación. Según Compañ (2020) debido a la pandemia no se puede pretender dar continuidad a los aprendizajes con equidad e inclusión debido a diversas inconsistencias desde diferentes ámbitos. Por ello es necesario buscar desarrollar nuevas formas de educar en distintos escenarios.

\section{Conclusiones}

En la actualidad existen diferentes definiciones sobre la epistemología en Política Educativa, pero cada país debe establecer su filosofía de su Política Educativa en base a su realidad y debe estar cimentada en fundamentos filosóficos e ideológicos.

La Política Educativa, estable las normas y leyes que orientan el accionar; o al modo en que se educa al ser humano en sociedad. Actualmente, la Política Educativa es inclusiva e igualitaria. Sin embargo, según la literatura es difícil platear una educación inclusiva. 
La Filosofía de la Educación, es un fundamento básico de las ideas educativas y pedagógicas que constituye referencia obligada de todos los profesores, directivos y asesores, como única vía para apreciar los modelos educativos que rigen en la educación, con el fin de formar al ser humano en su forma biopsicosocial.

Los fundamentos filosóficos en el orden educativo tratan del quehacer educativo a partir de la práctica educativa. Con respecto a lo ideológico, esta debe contemplar inclusión, diversidad cultural, igualdad de género, origen, lengua materna, religión para los alumnos y profesores.

\section{Conflictos de intereses}

Los autores firmantes del presente trabajo de investigación declaran no tener ningún potencial conflicto de interés personal o económico con otras personas $\mathrm{u}$ organizaciones que puedan influir indebidamente con el presente manuscrito.

\section{Contribuciones de los autores}

Preparación y ejecución: MB, RE, SA, AR; Desarrollo de la metodología: MB, RE, SA, AR; Concepción y diseño: MB, RE, SA, AR; Edición del artículo: MB, RE, SA, AR; Supervisión del estudio: $\mathrm{MB}, \mathrm{RE}, \mathrm{SA}, \mathrm{AR}$.

\section{Referencias}

Beuchot, M. (2015). La filosofía de la educación desde una hermenéutica analógico-icónica. Voces de la filosofía de la educación. Primera edición. Pág.

20. http://biblioteca.clacso.edu.ar/clacso/se/201710 13112316/Voces_filosofia_educacion.pdf

Compañ, J. (2020). Acciones de políticas educativas ante la emergencia sanitaria del Covid-19. Acciones de políticas educativas ante la emergencia sanitaria del Covid-19L(3):95-120

Glaser, Barney y Strauss, Anselm (1967). The discovery of grounded theory: strategies for qualitative research, Nueva York: Aldine.

Lewin, K. (1988). La teoría del campo en la ciencia social, Barcelona, España: Paidós Ibérica.
Martínez, F. (2018). Reflexiones sobre las políticas educativas. REVISTA LATINOAMERICANA de estudios educativos, nueva época, suj, 71-76.

Morales-Gómez, G., Reza-Suárez, L., GalindoMosquera, S., \& Rizzo-Bajaña, P. (2019). ¿Qué Significa "Fundamentos Filosóficos" De Un Modelo Educativo De Calidad? Ciencia Unemi, 12(31), 116-127. doi: 10.29076/issn.25287737vol12iss31.2019pp116-127p.

Morin, E. (1999). Los siete saberes necesarios para la educación del futuro, Paris, Francia: Unesco.

Muñoz, H. P., Lobos, A. C., Kawada, F. H., \& Buzzetti, D. M. (2020). Normocentrismo, Diversidad y Alteridades: Deconstruyendo las Políticas Educativas Inclusivas en Chile. Education Policy Analysis Archives, 28(1068-2341), 7-11. Recuperado de https://dialnet.unirioja.es/servlet/articulo?codig $\mathrm{o}=7759438$

Pérez, T. G. (2018). Equal educational policies in Spain. Gender equality in teaching studies. Education Policy Analysis Archives, 26(8). doi: 10.14507/epaa.26.2764

Rayou, P. y Van Zanten, A. (2015). Les 100 mots de l'education. París: Presses Universitaires de France.

Viennet, R. y Pont, B. (2017). Education policy implementation. A literature review and proposed framework. OECD Education Working Papers. París: OCDE.

Villegas, C., \& Cornieles, L. (2018). Ideological background of the concept of educational development during the first 6 years of the government of Hugo Chávez. Discurso y Sociedad, 12(2), 367-395. Recuperado de http://www.dissoc.org/ediciones/v12n02/DS12 $\% 282 \% 29$ Villegas\&Cornieles.pdf

Tello, C.; Mainardes, J. La posición epistemológica de los investigadores en política educativa: debates teóricos en torno a las perspectivas neomarxista, pluralista y posestructuralista. Archivos Analíticos de Políticas Educativas, v. 20, n. 9, p. 1-37, 2012. Disponible en: $<$ https://epaa.asu.edu/ojs/article/viewFile/988/9 42>. Acceso en el: 24 mar. 2013.

Percival (2017). Lo histórico y filosófico Fundamentos del conservador Política educativa. FORUM: para la educación integral, 59, Número 2, 1-9. 
Disponible

en

http://dx.doi.org/10.15730/forum.2017.59.2.273

Tello, C. (2019). Notas Históricas y Epistemológicas sobre el Campo de la Política. Educativa en Latinoamérica. Educ. Soc., Campinas, 40: e0205680. Doi: 10.1590/ES010173302019205680

Viennet, R. y Pont, B. (2017). Education policy implementation. A literature review and proposed framework. OECD Education Working Papers. París: OCDE. 